\title{
PROCESOS DE LA CIENCIA Y DESARROLLO COGNITIVO EN BACHILLERATO
}

\author{
PALACIOS, C., López, F., GARROTE, R. y MONTES, P. \\ I.B. «Cardenal Herrera Oria». Madrid.
}

\section{SUMMARY}

The study described in this report was designed to investigate the relationship between integrated process skills and formal thinking in high school students. Paper and pencil measurement of both variables was made using a sample of 189 students, the average age being, 15 years. The resulting correlation showed a Pearson's of 0.68 . An empirical analysis of the cognitive demand of different science process skills was carried out. Some implications for Science Education are, finally, discussed.

\section{INTRODUCCIÓN}

En las dos últimas décadas, diferentes investigadores en Enseñanza de las Ciencias han coincidido en considerar los llamados "procesos de la ciencia" como el principal objetivo de la instrucción científica en niveles de enseñanza no universitaria (Gagné 1965, Theory Into Action - A Guide to Science Curriculum Development 1964, Science Objetives 1969). De acuerdo con Finley (Finley 1983) los procesos de la ciencia se caracterizan, básicamente, por los siguientes rasgos peculiares:

a) Cada proceso es una destreza intelectual especifica empleada por los científicos y aplicable a la comprensión de un fenómeno.

b) Cada proceso se corresponde con una conducta identificable como típicamente cientifica que puede ser aprendida por los estudiantes.

c) Los procesos son transferibles a otros dominios de contenido y contribuyen al pensamiento racional sobre asuntos cotidianos.

Como consecuencia de tal planteamiento se han producido cursos y diseños curriculares enfocados primordialmente a desarrollar este tipo de destrezas (Science curriculum unprovement sutdy 1971; Intermediate Science curriculum study 1975, Science - A process aproach 1967). En nuestro país, la orientación oficial inicial de la Reforma de las Enseñanzas Medias ha pretendido poner también el acento en las habilidades típicas dei quehacer cientifico, a la hora de plantear un proyecto de currículo de ciencias (Hacia la Reforma
I. Documentos 1985). Independientemente de que trabajos recientes defienden un cierto equilibrio entre el enfoque basado en los conceptos y el enfoque basado en los procesos, en el diseno de los currículos científicos (Finley 1983, Pozo et al. 1986, Gil Pérez 1986), está claro que bajo la denominación de procesos de la ciencia se agrupan habilidades con un grado de dificultad y de exigencia intelectual muy diferente, por lo que parece deseable disponer de una orientación fundada respecto qué destrezas pueden ser incluidas y cuáles no en un curso dado. Diversas investigaciones han sido realizadas en otros paises, sobre la influencia de diferentes factores psicológicos en la adquisición de las destrezas cientificas (Padilla et al. 1983, Tobin et al. 1982), pero no existia ninguna información cuantitativa referida a alumnos de nuestro país.

En la presente investigación hemos analizado la influencia del nivel piagetiano de desarrollo cognitivo, medido mediante el test de Longeot, sobre la adquisición de seis habilidades cientificas diferentes, medidas con la versión española de TIPS (Test of the Integrated Science Process Skills), sobre una muestra de alumnos de Bachillerato que no fueron sometidos previamente a una instrucción especialmente dirigida a desarrollar este tipo de destrezas. Mediante un procedimiento empírico hemos determinado la demanda cognitiva de cada una de las habilidades consideradas. Tras presentar los resultados de un estudio correlacional complementario, algunas implicaciones de los resultados obtenidos respecto de la validez de TIPS, así como res- 
pecto de la incorporación de algunas de las destrezas analizadas como objetivos de los cursos de ciencias son, finalmente, discutidas.

\section{MÉTODOS}

- Muestra: La muestra, cuyo nivel socioeconómico puede ser considerado como medio/medio-alto, estuvo constituida por 189 sujetos (88 varones y 101 mujeres), la totalidad de los alumnos matriculados en $2^{\circ}$ de BUP en el I.B. Cardenal Herrera Oria durante el curso 83-84. A todos ellos se les administraron, a principio de curso, sendas versiones del Test de Longeot y del TIPS, respectivamente.

\section{- Pruebas:}

* Test de Longeot (TL): Es una prueba piagetiana de lápiz y papel que mide cuatro aspectos característicos del pensamiento formal (ver anexo):

- Inclusión de clase (TLI)

- Logica de proposiciones (TL2)

- Razonamiento combinatorio (TL3)

- Razonamiento probabilístico (TL4)

Se ha utilizado una versión adaptada al castellano por I. Aguirre de Cárcer cuya fiabilidad para nuestra muestra, determinada mediante la fórmula de KuderRichardson KR-21, es de 0,77 . Su validez ha sido analizada en investigaciones anteriores (Longeot 1962, Longeot 1965, Ahlawat et al. 1982, Ward et al. 1981, López Rupérez et al. 1986a).

* TIPS: Es un test de lápiz y papel que mide diferentes destrezas propias del quehacer científico (Gerald et al. 1980), las cuales han sido agrupadas en nuestro estudio en las siguientes categorías (ver anexo):

- Reconocimiento de variables (MC17)

- Identificación de hipótesis (MC2)

- Identificación de la definición operacional de una variable implícita en la descripcion de una investigación (MC31)

- Identificación de la definición operacional de una variable explícita en la descripción de una investigación (MC32)

- Reconocimiento de diseños experimentales (MC4)

- Reconocimiento de gráficas (MC5)

- Interpretación de gráficas (MC6)

Se ha utilizado la versión adaptada al castellano, (Vera et al. 1984) que ha resultado tener una fiabilidad para nuestra muestra de 0,67 utilizando la formula de KuderRichardson KR-21; dicho resultado puede compararse con el valor 0,87 del mismo parámetro obtenido por los autores de la aplicación sobre una muestra de 709 sujetos.

- Variables: En la investigación se han manejado las siguientes variables (Tabla I):
- MC: número de respuestas correctas para el TIPS

- TL: puntuación total en el test de Longeot

$-\operatorname{MCi}(i=1,2,31,32,4,5,6): \pi^{\circ}$ de respuestas correctas para cada parte del TIPS

- TLi $(\mathrm{i}=1 . .4)$ : puntuación obtenida en cada subtest de Test de Longeot

- NivC: estadio cognitivo en el que se encuentra el alumno. Para determinar el valor de esta variable y siguiendo el criterio empleado en investigaciones anteriores, se ha clasificado a un alumno en:

Concreto (NIVC $=1$ ) cuando se comporta como tal al menos en tres de los cuatro sub-tests del Test de Longeot

Formal consolidado $($ NIVC $=3$ ) cuando lo es para al menos tres de los cuatro sub-tests del Test de Longeot De transición (NIVC $=2$ ) cuando no está incluido ent ninguna de las dos categorías anteriores

- SETi ( $\mathrm{i}=1,2,31,32,4,5,6)$ : grado de adquisición de cada habilidad. Variable dicotómica que toma el valor 1 cuando el alumno responde correctamente, al menos, las dos terceras partes de los ítems que miden dicha habilidad, y el valor 0 en caso contrario.

- SEXO: Se ha asignado el valor 0 a los varones y el valor $I$ a las mujeres.

- Técnicas de análisis: Para la realización de esta investigación se ha utilizado el paquete de programas SPSS (Norman et al. 1975) disponible en el ordenador Digital VAX-VMS del Centro de Cálculo de la Universidad Autónoma de Madrid y, particularmente, los subprogramas CROSSTAB, FREQUENCIES, BREAKDOWN y PEARSON CORR, apropiados a las diferentes situaciones de análisis requeridos en la búsqueda de relaciones inicialmente planteadas.

Tabla I

Conjunto de las variables empleadas en la investigación junto con sus correspondientes rangos de variacion.

\begin{tabular}{|c|c|c|c|}
\hline YARIA8LES & Yhlores & Whathates & FLLOES \\
\hline il & $0-31$ & H. & $0-33$ \\
\hline It1 & 0.5 & $H 1$ & $0-9$ \\
\hline I12 & 0.7 & $4: 2$ & $0-9$ \\
\hline fis & 0.10 & $1 \times 31$ & $0-3$ \\
\hline \multirow[t]{2}{*}{ IL6 } & $0-9$ & NC32 & 0.3 \\
\hline & & $\sqrt{4}$ & $0-3$ \\
\hline nive & $1-3$ & wSS & $0-3$ \\
\hline \multirow[t]{8}{*}{$s \in \times 0$} & $0-1$ & $\mathrm{HC}_{6}$ & $0-3$ \\
\hline & & stit & $0-1$ \\
\hline & & $s \varepsilon+2$ & $0-1$ \\
\hline & & sets & $0-\uparrow$ \\
\hline & & SET32 & $0-1$ \\
\hline & & SEIS & $0-1$ \\
\hline & & seis & $a-1$ \\
\hline & & 5816 & 0.1 \\
\hline
\end{tabular}




\section{RESULTADOS Y DISCUSIÓN}

\section{a) And́lisis de la demanda cognitiva de las diferentes habilidades}

Con el proposito de analizar la demanda cognitiva de cada destreza (Shayer et al. 1984), hemos obtenido mediante el subprograma CROSSTAB la distribución porcentual de alumnos que al iniciar $2^{\circ}$ de BUP tienen adquirida cada habilidad cientifica en cada uno de los tres niveles cognitivos considerados, así como el porcentaje total respecto de la muestra globalmente considerada. Aunque una de las variables controladas ha sido el sexo, los resultados que se presentan a continuación son los obtenidos al considerar la muestra en su conjunto por no haber encontrado diferencias significativas entre varones y mujeres.

Por otra parte se ha determinado mediante el subprograma FREQUENCIES la distribución porcentual de la muestra sólo de niveles cognitivos dando como resultando un $9 \%$ de alumnos concretos, un $58 \%$ en transición, y un $33 \%$ de formales consolidados; cifras que coinciden con las descritas en trabajos anteriores (L $\delta$ pez Rupérez et al. 1986b) para una muestra diferente pero de análogas características de extensión, edad, sexo y extracción social.

A continuación se muestran y discuten los resultados obtenidos para cada una de las habilidades cientificas contempladas en el TIPS.

$\mathrm{MCl}$ : Reconocimiento de variables (dependiente, independiente, de control).

Los resultados que se representan en la figura 1 parecen mostrar una considerable exigencia intelectual para la habilidad científica $\mathrm{MCl}$, pues sólo el $24 \%$ de los alumnos formales y el $16 \%$ del total tienen adquirida dicha destreza. Por otra parte, la discriminación por niveles cognitivos es poco clara. El origen de tal circunstancia podría radicar en la forma en que se pretende evaluar dicha habilidad. En el test a los alumnos se les pregunta, literalmente, cuál es la «variable dependiente», la "variable independiente» y «la que se mantiene fija por el experimentador» y debido, muy

\section{figura $I$}

Estimación de la exigencia cognitiva de la destreza MCl (Reconocimiento de variables cientificas) expresada en términos de porcentajes de alumnos, que para cada uno de los tres niveles cognitivos, concreto, transición y formal (consolidado) tienen adquirida dicha destreza.

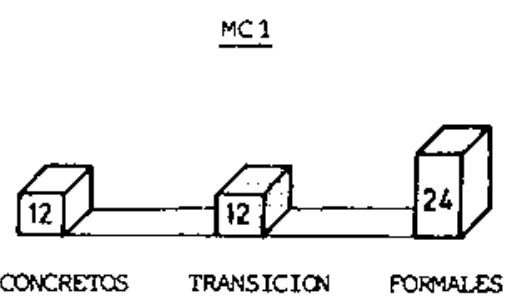

probablemente, al conocimiento insuficiente por parte de los sujetos del significado de los dos primeros términos se producen errores en las respuestas que no reflejan el nivel real de exigencia cognitiva de las destrezas correspondientes. Esta hipotesis es, en parte, confirmada por el hecho de que al estudiar aisladamente la distribución por porcentajes de la habilidad para reconocer la variable de control expresada en términos de «variable que el experimentador mantiene fija» responde correctamente a, como mínimo, dos de los tres items correspondientes a dicha habilidad, el $25 \%$ de los alumnos clasificados como concretos, el $36 \%$ de los considerados en transición y el $41 \%$ de los formales consolidados, es decir, un $37 \%$ del total.

El anterior análisis nos permite sugerir que siempre que se trate de medir este tipo de habilidades en alumnos no especialmente entrenados debiera utilizarse una terminología asequible. Así, por ejemplo, preguntar cuál es el factor que el experimentador modifica, o cuál es la variable causa es más comprensible para los alumnos que preguntar cuál es la variable independiente. Análogamente, el preguntar cuál es el efecto o consecuencia, como alternativa al empleo del término «variable dependientes, elimina el papel intermedio de la comprensión verbal de una jerga especializada que puede no estar disponible en este tipo de alumnos.

\section{$\mathrm{MC2}$ : Identificación de hipotesis.}

Los resultados representados en la figura 2 muestran que el nivel de desarrollo cognitivo discrimina fuertemente a los individuos en cuanto a la adquisición de esta habilidad. Por otra parte, sólo el $41 \%$ de los alumnos considerados son capaces, al comenzar $2^{\circ}$ de BUP, de identificar la hipótesis del investigador cuando se les proporciona la situación experimental correspondiente.

MC31 y MC32: Reconocimiento de definiciones operacionales.

Los autores del TIPS agrupan bajo esta denominación habilidades que en nuestro estudio han sido consideradas de forma separada como MC31 y MC32 y han dado lugar a resultados muy diferentes. Al analizar los

figura 2

Estimación de la exigcncia cognitiva de la destreza MC2 (Identificación de hipótesis).

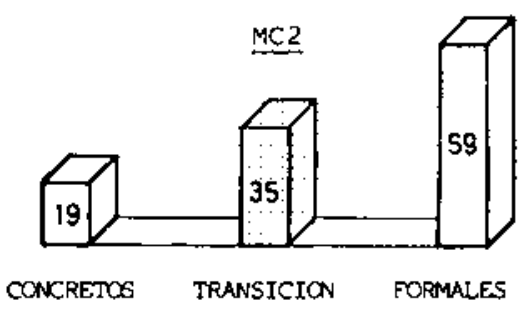


resultados de dicha prueba, observamos que los seis items, que según los autores miden la habilidad para reconocer definiciones operacionales, cuando se consideraban conjuntamente daban resultados un tanto anómalos, lo que nos llevó a analizar detenidamente sus enunciados respectivos advirtiendo en ellos diferencias apreciables en cuanto a su exigencia intelectual. El estudio cuantitativo fue entonces efectuado tras una reclasificación de los items correspondientes. Asi consideramos MC31 como la habilidad para reconocer la definición operacional de una variable implícita en la descripción de una investigación y MC32 como la habilidad para reconocer la definición operacional de una variable que aparece explicitamente referida en la descripción de una investigación.

Los resultados correspondientes a la habilidad MC31 se muestran en la figura 3 , y reflejan un nivel de discriminación por niveles cognitivos considerable así como un porcentaje de sólo un $39 \%$ de alumnos que tienen adquirida dicha habilidad.

\section{figura 3}

Estimación de la exigencia cognitiva de la destreza MC31 (Identificación de la definición operacional de una variable implícita en la descripción de una investigación).

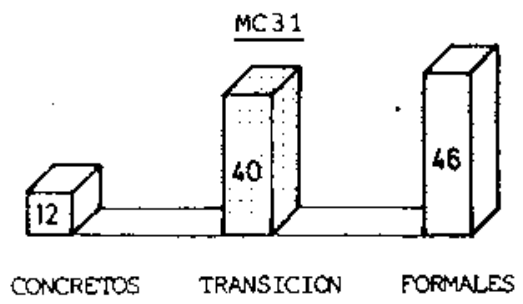

La figura 4 representa, por su parte, los resultados obtenidos para la habilidad MC32 que, como puede apreciarse, son completamente diferentes de los anteriores. Esta habilidad la poseen el $81 \%$ de los alumnos considerados como concretos, el $92 \%$ de los considerados en transición y el $98 \%$ de los formales consolidados, con un porcentaje global del $93 \%$, es decir, la casi totalidad de los alumnos que inician el $2^{\circ}$ de BUP.

\section{figura 4}

Estimación de la exigencia cognitiva de la destreza MC32 (Identifjcación de la definición operacional de una variable explícita en la descripción de una investigación).

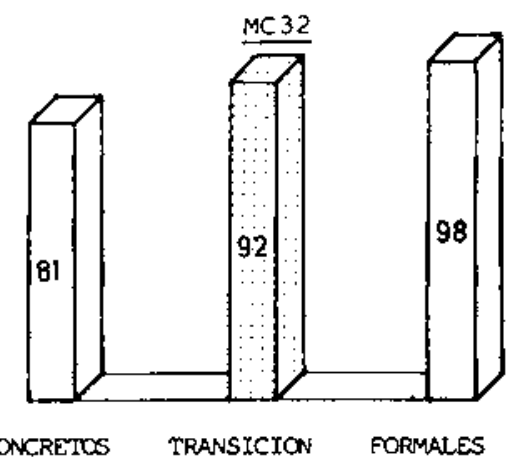

Las diferencias obtenidas en la actuación de los alum. nos entre ambos tipos de ítems sugieren la posibilidad de que sea el estilo cognitivo, y más concretamente la dimensión dependencia/inđependencia de campo (DIC) (Witkin et al. 1977), la variable que esté influyendo realmente en la discriminación observada ante la destreza MC31, más que el nivel de desarrollo cognitivo propiamente dicho. En este caso parte de la información relevante está enmascarada en el enunciado y dado que, como hemos demostrado en investigaciones anteriores (López Rupérez et al. 1986c), la validez del test de Longeot está limitada por el hecho de que el formato abierto de parte de sus ítems facilite la actuación de los sujetos independientes de campo frente a los dependien. tes, la distribución que se recoge en la figura 3 podría estar a fectada no sólo por el nivel de desarrollo cognitivo, sino también por el estilo cognitivo (DIC) del sujeto, operando a través de fa clasificación por niveles efectuada a partir del test de Longeot. De ahí que cuando se elimina el enmascaramiento en la referencia a la variable en cuestión y ésta aparece explícitamente en el enunciado (MC32) se anula prácticamente la discriminación por efecto del nivel de desarrollo cognitivo. Este resultado viene a sumarse a algunos otros (Linn et al. 1978) en el sentido de poner en evidencia la influencia del contexto en el que se efectúa una tarea sobre la actuación del sujeto ante ella.

MC4: Reconocimiento de disenos experimentales.

Como se muestra en la figura 5 esta habilidad la tie. nen adquirida el $31 \%$ de los alumnos concretos, el $75 \%$ los de transición y el $86 \%$ de los formales, lo cual, en términos globales, supone su adquisición, al nivel académico y de edad considerado, por el $75 \%$ de los alumnos de la muestra analizada.

\section{figura 5}

Estimación de la exigencia cognitiva de la destreza MCs (Reconocimiento de gráficas).

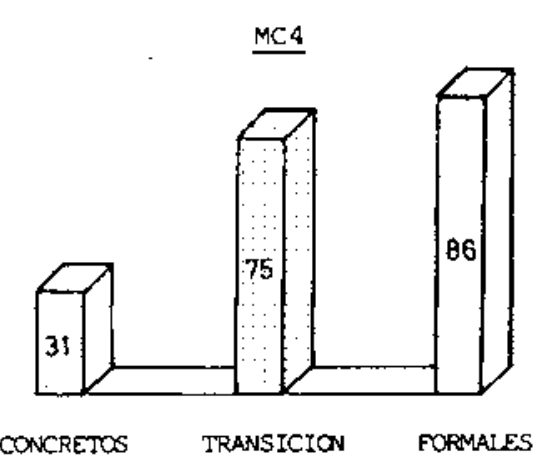

MC5: Reconocimiento de gráficas.

Los resultados representados en la figura 6 indican que el nivel de desarrollo cognitivo no guarda una clara relación con el porcentaje de sujetos que tienen adquirida la habilidad. Este hecho, en principio anómalo, pue- 
figura 6

Estimación de la exigencia cognitiva de la destreza MC5 (Reconocimiento de graficas).

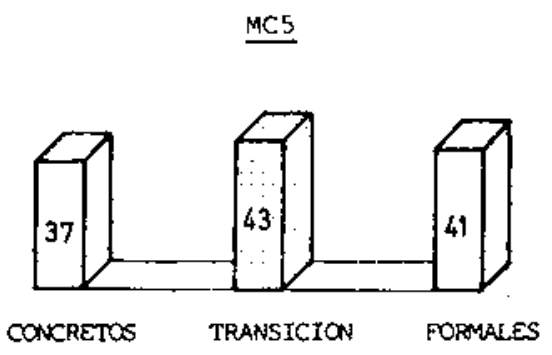

de explicarse de un modo similar al referido anteriormente para la destreza $\mathrm{MC1}$, apelando a la interferencia con el lenguaje. Así para evaluar la adquisición de la habilidad MCS se les propone a los alumnos que sobre cuatro gráficas dadas, identifiquen la que representa correctamente la información contenida en una tabla de valores (ver anexo). Esta habilidad está medida por tres items, dándose la circunstancia de que, en dos de éstos, uno de los distractores presenta la variable independiente en ordenadas y la dependiente en $a b$ cisas. La influencia del conocimiento, por parte del sujeto, de las normas de representación gráficas de datos experimentales se superpone, en este caso, a la de su nivel de desarrollo cognitivo.

MC6: Interpretación de gráficas.

En la adquisición de esta habilidad se aprecia claramente el efecto discriminatorio del nivel de desarrollo cognitivo, destacando un nivel de eficiencia del $100 \%$ en alumnos formales consolidados (fig. 7). A pesar de dicho efecto la destreza está adquirida por un $91 \%$ de la muestra completa. figura ?

Estimación de la exigencia cognitiva de la destreza MC6 (Interpretación de gráficas).

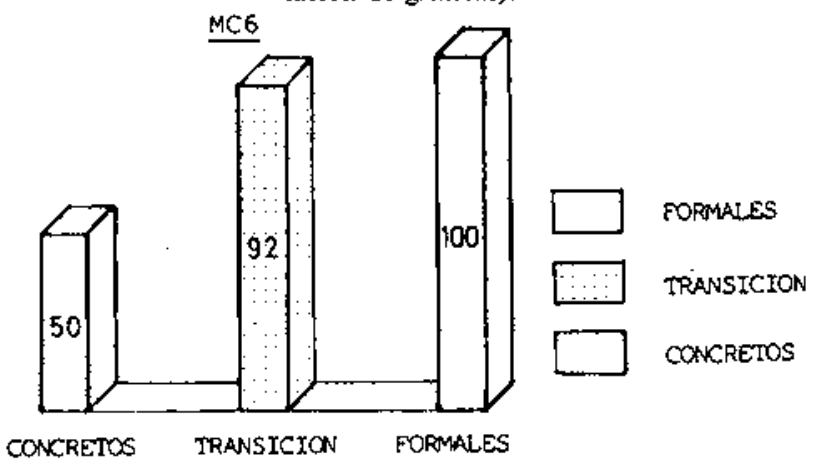

b) Análisis de la correlación razonamiento formalprocesos de la ciencia

Con la intención de complementar el estudio de las relaciones entre nivel de desarrollo cognitivo y procesos de la ciencia, hemos efectuado diferentes análisis de correlación entre la variable $T L$, considerada como una medida global del razonamiento formal del alumno, y cada una de las variables MCi correspondientes a las diferentes destrezas cientifícas consideradas. La tabla II muestra los resultados obtenidos por nosotros y, junto a ellos, los obtenidos por Padilla et al. (1983) utilizando como instrumento de medida del razonamiento formal el TOLT (Test of Logical Thinking) elaborado por Tobin y Capie (1980). Las cifras que figuran entre paréntesis son las que resultan tras la corrección efectuada en los coeficientes de correlación r de Pearson, para reducir la influencia debida a las limitaciones en la fiabilidad de las pruebas empleadas (Magnuson 1977). En nuestro estudio hemos medido dicha fiabilidad mediante la fórmula de Kuder-Richardson KR-21 (Magnuson 1977); aquellos valores para los cuales

Tabla II

Valores de los coeficientes de correlación de Pearson entre las variables $\mathrm{TL}$ y $\mathrm{MCl}$ y valores de fiabilidades de las prucbas.

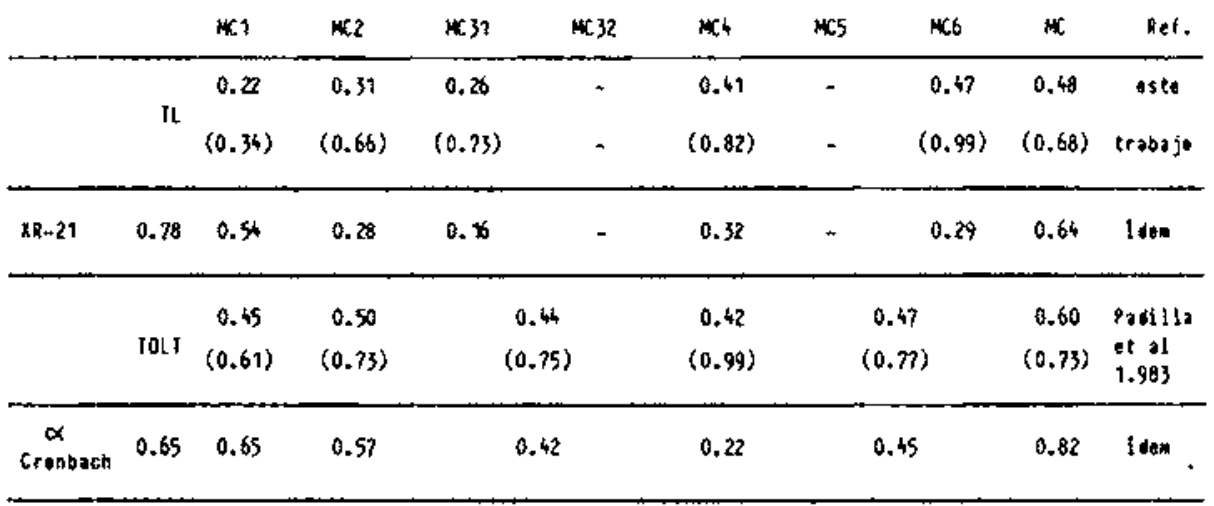

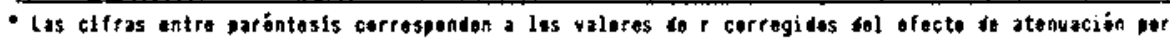
liaftactenes en lo fiabilicad a las aruebas.

MOTh: Las paráuetros KRa-27 y $\alpha$ e Cronbach son des paranetros analegos que aiden la fiabilivad de una gruaba on teralnas to consistencia internz (Magnuson, 1977). 
$\mathrm{p}<0.001 \mathrm{r}<0.200 \mathrm{KR}-21<0.15$ han sido elimina dos de la tabla.

El valor de la r de Pearson entre las variables TL y MC globalmente consideradas, corregido de los efectos de atenuación ya referidos, alcanza la cifra de 0.68 lo que pone de manifiesto por sí solo la fuerza de la relación entre ambas variables. El estudio, destreza por destre$\mathrm{za}$, indica que cuatro de ellas, a saber, la identificación de hipótesis (MC2), el reconocimiento de una defini. ción operacional (MC31), el reconocimiento de dise. nos experimentales (MC4) y la interpretación de gráficas (MC6) presentan un coeficiente de correlación corregida superior a un valor umbral de 0.5 ; dichas destrezas coinciden con las que se deducen de los datos de Padilla et al, (1983) cuando se eleva para ellos el umbral de $\mathrm{r}$ a 0,7 , valor éste razonable porque sus cifras son sistemáticamente mayores que las nuestras (ver tabla II). Por otra parte los resultados de estos análisis correlacionales son, en términos generales, con* gruentes con los obtenidos en el apartado anterior sobre la demanda cognitiva de las diferentes destrezas consideradas.

\section{c) Algunas implicaciones en la instrucción científica}

El estudio de las diferentes habilidades científicas analizadas desde el punto de vista de su demanda cognitiva ha puesto en evidencia problemas de validez de contenido para algunos de los ítems del TIPS; concretamente para los inciuidos en las habilidades representa. das por nosotros en la forma $\mathrm{MCl}$ (reconocimiento de variables) MC31 (identificación de definiciones operacionales) y MC5 (reconocimiento de gráficas). De las demás habilidades analizadas, la $\mathrm{MC} 32$ (identificación de definiciones operacionales) no discrimina de una for* ma notable entre sujetos formales, concretos y en transición, o to que es lo mismo, es suficientemente asequible aún para los alumnos concretos si la informa* ción relevante no está enmascarada; el resto, $\mathrm{MC2}$ (identificación de hipótesis), MC4 (reconocimiento de diseños experimentales) y MC6 (interpretación de gráficas) discriminan suficientemente entre los sujetos en función de su nivel de desarrollo cognitivo; no obstante, mientras que MC2 sólo está disponible como destreza adquirida para alrededor del $40 \%$ de los alumnos al comenzar $2^{\circ}$ de BUP; MC4 y MC6 lo están para más de las dos terceras partes de la muestra considerada.

La extensión de nuestros datos a la población está condicionada por el hecho de que el estatus socioeconómico de nuestra muestra sea medio/medio-alto. Debido a la reconocida influencia de dicha variable sobre el nivel de desarrollo cognitivo, es claro que las anteriores cifras deben ser consideradas como límites su* periores a la hora de ser generalizadas. No obstante, advierten sobre las precauciones a tomar a la hora de decidir qué tipo de destrezas científicas deben ser incluidas en los diferentes cursos de ciencias y de disenar las estrategias didácticas correspondientes.

Otra cuestión a destacar, en este orden de ideas, es el hecho de que los diferentes ítems del TIPS, por su carácter de prueba de lápiz y papel, sean de reconocimiento o de identificación y no de realización. Parece claro que idear un diseño experimental para una investigación determinada, por ejemplo, lleva consigo una exigencia intelectual mayor que reconocerlo como adecua. do cuando se presenta por escrito.

Finalmente, la importancia - puesta de manifiesto con toda claridad en relación con el reconocimiento de de. finiciones operacionales - de la influencia del enmascaramiento de la información relevante de un ítem dado, sobre su nivel de dificultad, introduce un punto de reflexión sobre la confección de pruebas y enunciados de problemas que podrian discriminar negativamente, en cursos en los que las asignaturas de ciencias son obligatorias, a aquella fracción de alumnos que presentan, como rasgo estable de su personalidad, un estilo cog. nitivo dependiente de campo.

\section{Agradecimientos}

Agradecemos a Isabel Brincones la colaboración prestada en la administración de las pruebas a los alumnos y en su cotrespondiente correccion. 


\section{REFERENCIAS BIBLIOGRÁFICAS}

AHLAWAT, K.S. y BILIEM, V.Y., 1982. The factor Structure of the Longeot test. A Mesure of Logical Thinking. Journal of Research in Science Teaching, Vol. 19(8), pp. 647-658.

FINLEY, F.N., 1983. Science Process. Journal of Research in Science Teaching, Vol. 20(1), pp. 47-54.

GAGNÉ, R., 1965. The psychological basis of Science. A process approach. (AAAS miscellaneous publication, 65-68 Washington $D C$ ).

GERALD DILLASHAW, F. y OKEY, J.R., 1980. Test of the Integrated Science Process Skills for Secondary Science Student. Science Education, Vol. 64(5), pp. 601-608.

GIL PÉREZ, D. 1986. La metodología cientifica y la enseñanza de las ciencias, una relaciones controvertidas. $E n$ sehanza de las Ciencias, Vol.4 (2), pp. 111-121.

LINN, C.M. y LEVINE, D.I., 1978. Adolescent Reasoning: Influence of Question formal and Type of Variables on Ability to Control Variables. Science Education, Vol. 62(3), pp. 377-388.

LONGEOT, F., 1962. Un éssai d'application de la psychologie genetique a la psychologie differentielle. Bulletin de l'Institute National d'Etude, 18, pp. 153-162.

LONGEOT, F., 1965. Analyse statisque de trois tests genetiques collectifs. Bulletin de /'Instifute National d'Étude, 20, pp. 219-237.

LOPEZ RUPÉREZ, F., PALACIOS, C., BRINCONES, I., GARROTE, R. y SÁNCHEZ, I., 1986a. Pensamiento formal y rendimiento en Fisica. Análisis de la validez del test de Longeot por referencia a tests de rendimiento. $\mathrm{En}$ seftanza de las Ciencias, Vol. 4(1), pp. 36-44.

LÓPEZ RUPÉREZ, F., PALACIOS, C., BRINCONES, I., GARROTE, R. y SÁNCHEZ, J., 1986b. Análisis de la evolución del nivel piagetiano de desarrollo cognitivo en alumnos de Bachillerato. Un estudio longitudinal. Revista de Psicología General y Aplicado. Aceptado y pendiente de publicación.

LÓPEZ RUPÉREZ, F., PALACIOS, C. y SÁNCHEZ, I., 1986c. Cognitive style and formal thinking. Influence of the field dependence-independence dimension on performance of written Piagetian tests through the item format. Remitido a Journal of Research in Science Teaching para su publicacion.

MAGNUSON, D., 1977. Teoria de los tests. (Trillas: México).

MEC, 1985. Hacia la Reforma I. Documentos. (Servicio de Publicaciones MEC. Madrid).

MORRISTOWN, N.J., SILVER BURDETT, 1975. Intermediate Science Curriculum Study.
NORMAN, H.N., HADLAI, C., JENKINS, I.G., STEINBRENNER, H. y BENT, D.H., 1975. Statiscal Package for the Social Sciences (SPSS). (McGraw Hill: New York).

PADILLA, M.J., OKEY, J.R. y GERALD, F., 1983. Journal of Research in Science Teaching, Vol. 20(3), pp. 239-246.

POZO, J.I. y CARRETERO, M., 1986. Desarrollo cognitivo y aprendizaje escolar. Infancia y aprendizaje.

Science-A process aproach, 1967. AAAS Miscellaneous $\mathrm{Pu}$ blication 67-11.

Science curriculum unprovement study, 1971. (Rand McNally and $\mathrm{Co}$ : Chicago).

Science Objetives, 1969. Committee on Assesing the Process of Education, National Assessment of Educational Progress. Ann. Arbor, Mich.

SHAYER, M. y ADEY, P., 1984. La ciencia de ensentar ciencias. Desarrollo cognoscitivo y exigencias del currículo. (Narcea: Madrid).

Theory Into Action - A Guide to Science Curriculum Development, 1964. Washington D.C. National Science Teachers Association.

TOBIN, K. y CAPIE W., 1980. The development and validation of a group test of Logical thinking. Articulo presentado a la convención anual de la American Educational Research Association. Boston.

TOBIN, K, y CAPIE W., 1982. Relationships between formal reasoning ability, locus of control, academic engagement and integrated process skill achievement. Journal of research in Science Teaching, Vol. 19, pp. 113-121.

VERA, J.J., BRINCONES, I., POSADA, M.I., CAÑAS, A., PALACIOS, C., SASTRE, C., GOMEZCARRILLO, R., FERNANDEZ VALMAYOR, A., y AGUIRRE DE CARCER, I., 1982. Las destrezas del quehacer cientifico. Desarrollo de una prueba para evaluarlas. I Jornadas de Investigación didáctica en Fisica y Quimica. (ICE Universidad de Valencia).

WARD, C.R., NURRENBERN, S.C., LUCAS, S. y HERRON, P.W., 1981. Evaluation of the Longeot test of cognitive development. Journal of Research in Science Teaching, Vol. 18(2), pp. 123-130.

WITKIN, H.A., MOORE, C.A., GOODENOUGH, D.R. y COX, P.W., 1977. Field-Dependent and FieldIndependent cognitive style and their educational implications. Review of Educational Research, Vol. 47(1), pp. $1-64$. 


\begin{abstract}
ANEXO
Ejemplos de los items correspondientes a los diferentes subtests de las dos pruebas empleadas en la investigacion.
\end{abstract}

TL1

Escribe todas las paiabras que se pueden formar con las 4 letras: A,T,I,R y subraya aquéllas que tengan algún sentido en castellano.

TL2

Lee los siguientes razonamientos y subraya la conclusión exacta:

* Razonamiento:

- Armando es más ágil que Bernardo

- Bemardo es más ágil que Daniel

* Conclusiones:

- Bernardo es el más ágil de tos tres niños

- Ammando es el más ágil de los tres niños

- No podemos saberlo

\section{TL3}

En la planta baja de un edificio nuevo se van a abrir 4 comercios. Una panadería, una carnicería, un ultramarinos y una tibrería desean instalarse en esos locales. Cada uno de ellos puede elegir cualquiera de los 4 locales. ¿Cuáles son todas las formas posibles en que se pueden ocupar esos 4 locales?

\section{$\underline{\mathrm{TL} A}$}

En un garaje se guardan 24 vehículos: 4 camionetas y 20 coches de turismo. En un segundo garaje hay 54 vehículos: 9 camionetas y 45 coches de turismo. En un tercer garaje, encontramos 36 vehículos: 6 camionetas y 30 coches de turismo. ¿De cuál de los garajes tenemos más posibilidades de ver salir la primera camioneta?

a) Del lercer garaje ya que tiene más camionetás que el primer garaje y menos coches de turismo que el segundo.

b) Del segundo garaje, ya que es el que tiene más camionetas.

c) Del primer garaje, ya que es el que tiene menos coches de nurismo.

d) De cualquiera de los tres garajes, ya que los tres tienen el mismo número de camionetas con respecto al númeto total de vehículos estacionados.

\section{$\underline{\mathrm{MCl}}$}

Sara quiso comprobar si la temperatura tiene algún efecto sobre el crecimiento del moho del pan. Para ello preparó un cultivo de moho en 9 recipientes que tenían la misma cantidad y el mismo tipo de sustancias nutritivas. Mantuvo 3 recipientes a $0^{\circ} \mathrm{C}$. otros 3 a $90^{\circ} \mathrm{C}$ y otros 3 a la temperatura ambiente (unos $17^{\circ} \mathrm{C}$ ). Después de cuatro días examinó los recipientes y tomó nota del crecimiento del moho.

\section{- El factor que el experimentador se preocupa de mantener} constante es:
a) La temperatura de los recipientes
b) El tipo de recipiente utilizado
c) La tempesatura del moho
d) La cantidad de moho

- La variable dependiente es:
a) EI crecimiento del moho
b) La cantidad de sustancias nutritivas en cada recipiente
c) La temperatura de los recipientes
d) El número de recipientes a cada temperatura

- La variable independiente es:

a) La temperatura de los recipientes

b) La cantidad de sustancias nutritivas en cada recipiente

c) El crecimiento del moho

d) EI número de recipientes a cada temperatura

\section{$\mathrm{MC2}$}

¿Cuál es su hipótesis?

a) El tipo de sustancias nutritivas que se han usado producirá diferencias en de moho de pan.

b) La cantidad de moho es afectada por la temperatura.

c) La cantidad de moho está determinada por de sustancia nutritiva que se ha tulizado.

d) El número de recipientes influye en de moho.

\section{MC31}

Algunos alumnos estudian las condiciones de thuminación para las plantas. Eligen 12 plantas de la misma altura. Colocan 6 plantas en una ventana, soleada y oras 6 en una habitación oscura.

La altura de la planta se mide a diario durante tres semanas. También se miden la temperatura y la humedad y se anotan a diario. Todas las plantas se riegan de forma regular. Después de una semana las plantas que están en la habitación oscura parecen enfermas.

En este cxperimento, el crecimiento de las plantas se detemina así:

a) Contando el número de hojas de cada planta.

b) Midiendo las condiciones ambientales tales como la temperatura y la humedad.

c) Midiendo la distancia desde la superficie de la tierra hasta lo más aito de la planta.

d) Midiendo el intervalo de tiempo durante el cual las plantas están expuestas a la luz.

\section{MC32}

Una clase está estudiando el efecto del ejercicio físico en la frecuencia del pulso. Los alumnos ejecutart varias pruebas de salto y después miden el pulso. Un grupo salta durante un minuto. E! siguiente grupo salta durante dos minutos. Un tercer grupo salta durante tres minutos y un cuarto no salta.

¿Cómo medirías la frecuencia del puiso en este estudio?:

a) Contando el número de saltos por minuto.

b) Contando el número de latidos del corazón por minuto.

c) Contando el número de saltos que hace cada grupo. 
ANEXO

(Continuació)

\section{$\underline{\mathrm{MC} 4}$}

Juan se pregunta qué es lo que infuirá en el tiempo que tardan los cubitos de hielo en derretirse. Piensa que los factores que pueden influir en el tiempo de fusión son: el tamaño de los cubitos, la temperatura ambiente y la forma de los cubitos. Finalmente se decide a comprobar la hipótesis de que la forma de los cubitos afecta al tiempo que tarda en fundirse. $i Q u e ́$ diseño debe seleccionar Juan para comprobar su hipótesis?

a) Usar 5 cubitos, cada uno de forma y peso diferente. Utilizar otros tantos recipientes, todos a la misma temperatura. $\mathrm{Ob}$ servar el tiempo que tardant en derretirse los cubitos.

b) Usar 5 cubitos, todos con la misma forma, pero con pesos diferentes. Utilizar 5 recipientes idénticos, todos a la mis. ma temperatura. Observar ei tiempo que tardan en derretirse.

c) Utilizar 5 cubitos, todos del mismo peso, pero de formas diferentes. Utilizar 5 recipientes idénticos todos a la misma temperatura. Observar el tiempo que tardan en derretirse.

d) Usar 5 cubitos idénticos, todos del mismo peso, peto cada uno con forma diferente a los demás. Utilizar 5 recipientes idénticos con temperaturas diferentes. Observar el tiempo que tardan en derretirse.

\section{MC5}

El Sr. Gómez lleva a cabo unos experimentos en su huerto.

Pone cantidades diferentes de abono mineral en el suelo para ver como afecta esto a la producción de patatas. Este año tiene cuatro parcelas de patatas. Todas las parcelas tienen el mismo tamaño, pero cadauna de ellas recibe distintas cantidad de abono. La información que recoge está resumida en la siguiente tabla:

\begin{tabular}{|c|c|c|}
\hline Parcelia & 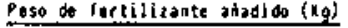 & Peso do patatas protucidas (kg) \\
\hline 1 & 0 & 47 \\
\hline 2 & 50 & 68 \\
\hline 3 & 150 & $\%$ \\
\hline 4 & 100 & 160 \\
\hline
\end{tabular}

¿Cuál de estas gráficas representa correctamente la inforrnación deł Sr.Gómez?
)

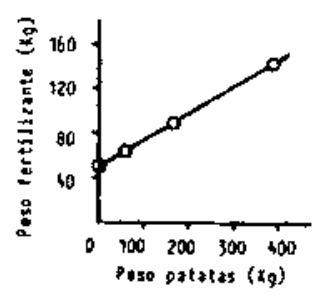

b)

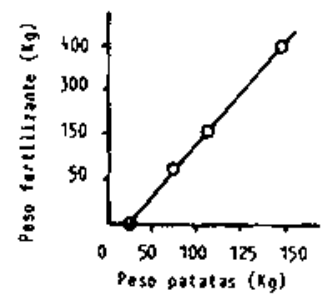

c)

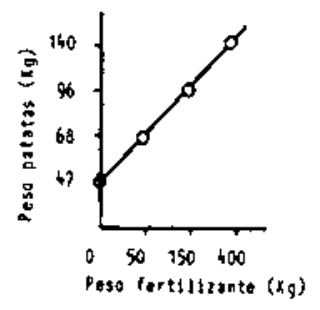

d)

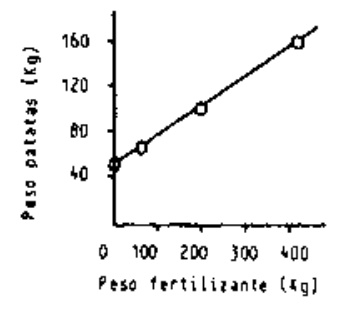

\section{$\underline{\mathrm{MC} 6}$}

En un campeonato de arco se mide el promediode flechas que hacen diara en el blanco. Las flechas se lanzan a diferentes distancias del blanco. Los datos están representados en el gráfico que sigue:

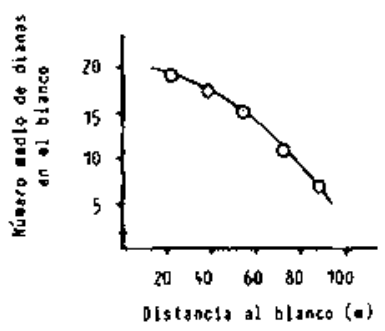

¿Cuál de los siguientes enunciados establece conectamente la relación entre las variables?

a) A medida que aumenta la distancia al blanco, aumenta el número de dianas.

b) A medida que aumenta cl número de dianas, aumenta la distancia al blanco.

c) A medida que aumenta la distancia al blanco. disminuye el número de dianas.

d) A medida que disminuye el número de dianas, disminuye la distancia al blanco. 